\title{
RESEARCH OF THE ATMOSPHERIC CORRECTION METHOD BASED ON APPROXIMATE SOLUTION OF MODTRAN TRANSMITTANCE EQUATION
}

\author{
A.M. Belov, V.V. Myasnikov \\ Samara National Research University, Samara, Russia
}

\begin{abstract}
The paper presents an experimental research of proposed method of atmospheric correction of hyperspectral remote sensing data. The method is based on approximate solution of MODTRAN transmittance equation using simultaneous analysis of remote sensing hyperspectral image and an ideal hyperspectral image of the same territory. Results of experimental research imitating conditions of practical method usage are presented.
\end{abstract}

Keywords: hyperspectral image, remote sensing data, transmittance equation, least square method, MODTRAN

Citation: Belov AM, Myasnikov VV. Research of the atmospheric correction method based on approximate solution of modtran transmittance equation. CEUR Workshop Proceedings, 2016; 1638: 256-262. DOI: 10.18287/16130073-2016-1638-256-262

\section{Introduction}

Atmospheric correction is one of the important stages of remote sensing data preprocessing especially in the case when the image analysis is based on the detected radiance spectral components $[1,2,3]$. The difference between sensor detected radiance and true surface radiance depends on many factors: solar declination, position of satellite vehicle, imaging angle, composition and moisture of atmosphere, etc. All these factors are taken into account in the generalized transmittance equation [4]. However, even with such detailed model of the atmosphere, it is difficult to solve precisely the problem of atmospheric correction because of the large number of unknown parameters which require a significant number of surface and meteorological measurements. Furthermore, the observed surface is not Lambertian usually and it is required to simulate a bidirectional reflectance distribution function for such surface reflectance modeling, which also requires laboratory studies of the structural and optical properties of materials [4].

There are a lot of methods and algorithms, that are used for the atmospheric correction of hyperspectral images: Scene-Based Empirical Approaches [5-8], Radiative Transfer Modeling Approaches [9-11] and Hybrid Approaches [12-16]. The paper 
presents an experimental research of the method of atmospheric correction of remote sensing hyperspectral images $[15,16]$ which belongs to the hybrid approaches group. The method is based on approximate estimation of parameters of simplified MODTRAN transmittance equation. There is no need to model the atmosphere explicitly with this approach, the problem reduces to the determination of the unknown coefficients of the equation, and all the details of light passing through the atmosphere remain in the model. However, such a solution requires an ideal (i.e. free from atmospheric distortions) hyperspectral image of the same territory. There is provided to use low-flying airborne hyperspectral imaging or ground station hyperspectral imaging. It is obvious that spatial resolution of the ideal image differs from the spatial resolution of the distorted image, moreover a set of ideal images overlaps the distorted image incompletely in common case. These facts require additional research.

\section{Method overview}

In the MODTRAN model the following simplification of transmittance is used [17]:

$$
L(i, j)=\frac{A \rho(i, j)}{1-\rho_{e}(i, j) S}+\frac{B \rho_{e}(i, j)}{1-\rho_{e}(i, j) S}+L_{a}
$$

where $i=1 \ldots M, j=1 \ldots N$ are coordinates in image plane, $L(i, j)$ - detected radiance, $\rho(i, j)$ - target pixel reflectance, $\rho_{e}(i, j)$ - surrounding pixels reflectance, $L_{a}$ - radiance of the atmosphere backscattering, $A, B$ - coefficients which depend on atmospheric and geometry condition, $S$ - atmospheric spherical albedo.

In common case values of $A, B, S, L_{a}$ are calculated by the MODTRAN model [18], however for the accurate calculation of these parameters it is necessary to have a model of the atmosphere consistent to the place, time, and weather conditions of imaging. In the absence of necessary parameters of the atmosphere model it is possible to perform atmospheric correction by an approximate solution of equation (1).

From each pixel of the ideal image we can define $\rho(i, j)$ and $\rho_{e}(i, j)$ by averaging within processing window. From atmospherically distorted image we can define $L(i, j)$. Thus, it is necessary to determine the four unknown parameters: $A, B, S, L_{a}$. The equation (1) is quadratic respective to the unknown parameters. However by fixing a certain value $L_{a}=L_{a}^{*}$ we proceed to linear equation respective to the unknown parameters $A, B, S$. Thus, writing equation (1) for each pair of corresponding pixels of ideal and source HSI, we have an overdetermined system of $M N$ linear equations with three unknowns $A, B, S$. 


$$
\left\{\begin{array}{l}
A \rho(1,1)+B \rho_{e}(1,1)+S \rho_{e}(1,1)\left(L(1,1)-L_{a}^{*}\right)=L(1,1)-L_{a}^{*} \\
A \rho(1,2)+B \rho_{e}(1,2)+S \rho_{e}(1,2)\left(L(1,2)-L_{a}^{*}\right)=L(1,2)-L_{a}^{*} \\
\cdots \\
A \rho(M, N)+B \rho_{e}(M, N)+S \rho_{e}(M, N)\left(L(M, N)-L_{a}^{*}\right)=L(M, N)-L_{a}^{*}
\end{array} .\right.
$$

To determine the unknown parameters it is proposed to use the method of least squares.

A fixed value of the parameter $L_{a}=L_{a}^{*}$ is determined in accordance with assumption that the value must be in interval $\left[0, \min L_{a}(i, j)\right]$. Further, when iterating the interval with the step $\Delta L_{a}$, for each value $L_{a}^{*}=k \Delta L_{a}, k \in \mathrm{N}$ is necessary to find a solution $x^{*}=\left[\begin{array}{lll}A & B & S\end{array}\right]^{T}$ of (2) and then choose the value of $L_{a}^{*}=k \Delta L_{a}, k \in \mathrm{N}$ that minimizes the error.

Atmospheric correction of distorted image is performed according to the formula [17]:

$$
\rho=\frac{L(i, j)-L_{a}+\frac{A}{B}\left(L(i, j)-L_{e}(i, j)\right)}{A+B+\left(L_{e}(i, j)-L_{a}\right) S}
$$

where $L_{e}(i, j)$ - spatial averaging of the detected radiance, and the meaning of the other parameters is similar to the equation (1).

The quality criterion of atmospheric correction based on a comparison of the ideal and the corrected image which applied in experimental research is calculated as:

$$
\varepsilon=\frac{1}{K} \sum_{k=1}^{K} \varepsilon_{k}
$$

where $\varepsilon_{k}$ - quality criterion for the certain spectral channel which is defined by the following expression:

$$
\varepsilon_{k}=\frac{\sum_{i=1}^{M} \sum_{j=1}^{N}\left(\widetilde{I}_{k}(i, j)-\hat{I}_{k}(i, j)\right)^{2}}{\sum_{i=1}^{M} \sum_{j=1}^{N} \widetilde{I}_{k}(i, j)^{2}}
$$

where $\tilde{I}_{k}(i, j)$ - sample of the ideal image, $\hat{I}_{k}(i, j)$ - sample of the corrected image. In fact, the above mentioned criterion is the averaging of the standard deviation normalized to the dynamic range of the channel of ideal image. 


\section{Experimental results}

We assume that ideal images and remote sensing data are obtained from different sources and spatial resolution of ideal images is higher and a set of ideal images overlaps a distorted image incompletely. Some experiments that imitate such conditions were carried out. Hyperspectral image JasperRidge98av.img obtained from AVIRIS aircraft was used as a source atmospherically-distorted image and the same image corrected by FLAASH algorithm was used as an ideal image.

To research the efficiency of the algorithm in conditions when the spatial resolution of source and ideal images differs following experiments were carried out:

A $256 * 256$ pixels fragment of the ideal image was expanded by a factor of 2, 3 and 4 . An expansion was performed by pixel duplicating. In a similar way the ideal image fragment was contracted by a factor of 2 and 4 . Contraction was performed by pixel averaging. Parameters of the transmittance equation were calculated using contracted and expanded fragments. Atmospheric correction of the source image was performed using calculated parameter sets. The quality criterion $\varepsilon$ was calculated for corrected images. Dependency of quality criterion $\varepsilon$ from expansion and contraction factor $E$ presented on figure 1.

Presented graphics show that quality criterion varies slightly for an expansion case and significantly for a contraction case. Thus we can conclude that proposed method can satisfactory be used when spatial resolution of the ideal image is higher that spatial resolution of source image and cannot be used in contrary.

a)
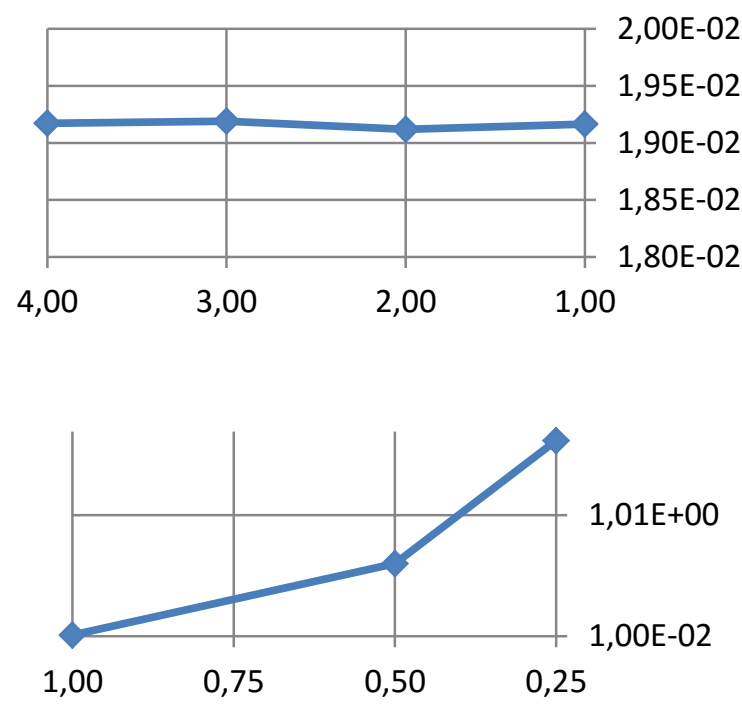

b)

Fig. 1. Dependency of quality criterion $\varepsilon$ from expansion and contraction factor $E$ : a) expansion, b) contraction 
To research an influence of percent of overlapping of source and ideal images $S$ following experiments were carried out:

Fragments of the ideal image that overlaps from 10 to 90 percent of source image in horizontal and vertical way were obtained. Parameters of the transmittance equation were calculated using these fragments. Atmospheric correction of the source image was performed using calculated parameter sets. The quality criterion $\varepsilon$ was calculated for corrected images. Dependency of quality criterion $\varepsilon$ from overlapping percent $S$ for a both cases presented on figure 2 .

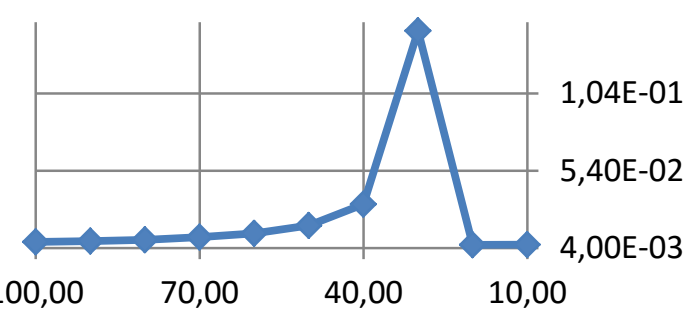

a)

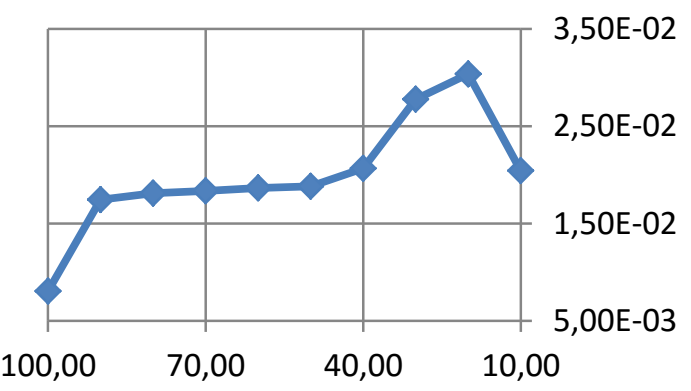

b)

Fig. 2. Dependency of quality criterion $\varepsilon$ from overlapping percent $S:$ a) vertical slicing, b) horizontal slicing

Significant increasing of atmospheric correction quality for overlapping percent 20 and 30 gave us reason to carry out additional experiments. Dependency of quality criterion for $\varepsilon_{k}$ from overlapping percent $S$ was studied for certain spectral channels. Experiment showed that such unexpected local quality increasing is typical for spectral channels 144-224 (fig $3 \mathrm{~b}$ ), for channels 1-143 dependency is monotonic increasing (fig 3 a).

Further statistical analysis of the ideal image showed that channels 144-224 contain a number of outliers affecting on parameters estimation and on atmospheric correction quality respectively. This fact complains that we obtain a better quality while use a reduced amount of source data. In this context there is a problem of increasing robustness of the method of atmospheric distortion parametric estimation. Since least 
squares method is high sensitive to outliers the idea for futher modification is to use a special preprocessing algorithm in order to adjust data.

\section{Conclusion}

Experimental research showed that proposed method is applicable for practical usage in atmospheric correction problem. Difference between reflectance values for ideal and corrected images for some spectral channels showed that proposed method requires a further modification aimed to adjust source data.

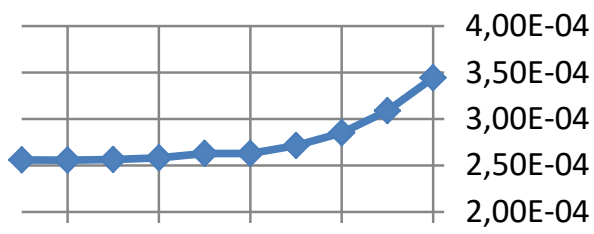

a)
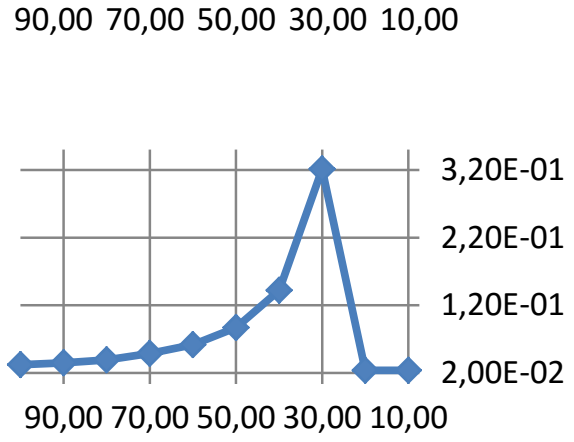

b)

Fig. 3. Dependency of quality criterion $\varepsilon_{k}$ from overlapping percent $S$ for vertical slicing: a) spectral channel 20, b) spectral channel 180

\section{Acknowledgements}

This work was financially supported by the Russian Scientific Foundation (RSF), grant no. 14-31-00014 "Establishment of a Laboratory of Advanced Technology for Earth Remote Sensing"

\section{References}

1. Denisova AYu, Myasnikov VV. Anomaly Detection for Hyperspectral Imaginery. Computer Optics, 2014; 38(2): 287-296. [in Russian] 
2. Denisova AYu, Myasnikov VV. Algorithms of Linear Spectral Mixture Analysis for Hyperspectral Images Using Base Map. Computer Optics, 2014; 38(2): 297-303. [in Russian]

3. Kuznetsov AV, Myasnikov VV. A comparision of algorithms for supervised classification using hyperspectral data. Computer Optics, 2014; 38(3): 494-502. [in Russian]

4. Schowengerdt R. Remote Sensing: Models and Methods for Image Processing (3rd Edition), Academic Press, 2006.

5. Chavez PS. An improved dark-object subtraction technique for atmospheric scattering correction of multispectral data. Remote Sensing of Environment, 1988; 24: 459-479.

6. Conel JE, Green RO, Vane G, Bruegge CJ, Alley RE. AIS-2 radiometry and a comparison of methods for the recovery of ground reflectance. Proceedings of the 3rd Airborne Imaging Spectrometer Data Analysis Workshop, 1987; 18-47.

7. Bernstein LS, Jin X, Gregor B, Adler-Golden S. Quick Atmospheric Correction Code: Algorithm Description and Recent Upgrades. Optical Engineering, 2012; 51(11): 111719-1 111719-11.

8. Kruse FA. Use of airborne imaging spectrometer data to map minerals associated with hydrothermally altered rocks in the northern Grapevine Mountains, Nevada and California. Remote Sens. Env., 1988; 24: 31-51.

9. Gao BC, Heidebrecht KB, Goetz AFH. Derivation of scaled surface reflectances from AVIRIS data. Remote Sens. Env., 1993; 44: 165-178.

10. Gao BC, Davis CO. Development of a line-by-line-based atmosphere removal algorithm for airborne and spaceborne imaging spectrometers. SPIE Proceedings, 1997; 3118: 132141.

11. Wu J, Wang D, Bauer ME. Image-based atmospheric correction of QuickBird imagery of Minnesota cropland. Remote Sensing of Environment, 2005; 99: 315-325.

12. Goetz AFH, Heidebrecht KB, Kindel B, Boardman JW. Using ground spectral irradiance for model correction of AVIRIS data. JPL Summaries of the 7th Annual Airborne Earth Science Workshop, 1998; 98-1: 159-168.

13. Clark RN, Swayze GA, Heidebrecht KB, Green RO, Goetz AFH. Calibration to surface reflectance of terrestrial imaging spectrometry data: Comparison of methods. Summaries of the 5th Annual JPL Airborne Earth Science Workshop, 1995; 95-1: 41-42.

14. Ardouin JP, Ross V. Methods for in-scene atmospheric compensation by endmember matching. U.S. Patent No. 20,150,161,768. 11 Jun. 2015.

15. Belov AM, Myasnikov VV. Atmospheric correction of hyperspectral images using approximate solution of MODTRAN transmittance equation. Computer Optics, 2014; 38(3): 489493. [in Russian]

16. Belov AM, Myasnikov VV. Atmospheric correction of hyperspectral images based on approximate solution of transmittance equation. Proc. SPIE 9445, Seventh International Conference on Machine Vision (ICMV 2014), 2015; 94450S: S-1-5. DOI:10.1117/ 12.2181364

17. Yuanliu X, Runsheng W, Shengwei L, Suming Y, Bokun Y. Atmospheric correction of hyperspectral data using MODTRAN model. SPIE Proceedings, 2003; 7123.

18. Kneizys FX, Robertson DC, Abreu LW, Acharya P, Anderson GP, Rothman LS, Chetwynd JH, Selby JEA, Shettle EP, Gallery WO, Berk A, Clough SA, Bernstein LS. The MODTRAN 2/3 Report and LOWTRAN 7 MODEL, Ontar Corporation, 1996. 